\title{
XLII. On professor Müller's account of the reflex function of the spinal marrow
}

\author{
Marshall Hall M.D. F.R.S.
}

To cite this article: Marshall Hall M.D. F.R.S. (1837) XLII. On professor Müller's account of the reflex function of the spinal marrow, Philosophical Magazine Series 3, 10:60, 187-193, DOI: 10.1080/14786443708649118

To link to this article: http://dx.doi.org/10.1080/14786443708649118

册 Published online: 01 Jun 2009.

Submit your article to this journal

Џ Article views: 2

Q View related articles $₫$ 
rific rays, and that the introduction of a concave lens tends to achromatize and improve the light. Our solar and oxy-hydrogen microscopes, therefore, instead of being used for purposes of amusement only, and limited to the exhibition of objects which are not affected easily by heat, may henceforward be employed for purposes of scientific investigation, and thereby assume the more important rank of valuable philosophical instruments.

Peckham, Feb. 10, 1837.

XLII. On Professor Mülden's Account of the Reflex Function of the Spinal Marrow. Communicated by Ma RSHALL HaLL, M.D., F.R.S., \&c.

[Continued from p. 129, and concluded.]

“DR. MARSHALL HALL distinguishes four kinds of muscular contraction: I st, The voluntary, which appears to depend on the brain; $2 \mathrm{nd}$, The respiratory*, which appears to depend on the medulla oblongata; 3rd, The involuntary, which depends on the nerves and muscles, and requires the immediate application of stimuli to the muscles provided with nerves, or to their nerves; and 4th, The reflecting, which continues, in part, after the voluntary and respiratory have ceased, and is connected with the medulla spinalis. It ceases after removal of the spinal marrow, though irritability does not diminish. In this fourth the motor stimulus does not originate in a central part of the nervous system, but at some distance from the centre; it is neither voluntary nor direct in its course, but rather excited by peculiar stimuli, which act, not immediately on the muscular fibres and motor nerves, but on membranous expansions, from which the stimulus is conducted to the medulla spinalis. Dr. Marshall Hall illustrates the importance of this reflecting function of the medulla oblongata and spinal marrow by some instances. The prehension of food is a voluntary act, and cannot be performed after removal of the brain ; the passage of the morsels of food over the glottis and through the pharynx depends on the reflex function, and still continues after the brain is removed. Although, for instance, the muscles which are active in this case, may also act voluntarily, yet the presence of the morsel in the pharynx produces a series of violent motions, which have been described above (p. 479.), and which arise from the stimulus of the morsel

* I am now of opinion that respiration itself is a part of the reflex or excito-motory function, and dependent upon appropriate excitor nerces.M. H. 
acting on the sensitive mucons nembrane, and this perception exciting the medulla oblongata to discharge in the motor nerves. Dr. Marshall Hall regards the further act of deglutition in the œsophagus as the effect of the stimulus acting immediately on the muscular fibres of the cesophagus, and the result of the irritability of the latter, which may appear very doubtful*. Even in beheaded young animals we may, however, as already shown, observe the reflected motorial excitement still following mechanical stimulation of the pharynx. Dr. Marshall Hall next shows the permanent influence of this function in the sphincters. 'The sphincter ani remains closed in a tortoise after decapitation, so long as the lower part of the spinal marrow is uninjured, but instantly becomes flaccid and opens, when the spinal marrow is removed.

"Dr. Marshall Hall divided the spinal marrow in a live $C_{0-}$ inber natrix between the 2 nd and 3rd vertebra. The motions ceased at once, and when the animal was not stimulated it remained quiet. But if it were stimulated, it continued moving for a long time; for at every altering position new parts of its surface came in contact with the ground: gradually it again became quiet, but the slightest touch again renewed the motion.

"Dr. Marshall Hall shows very beautifully the relation of the voluntary, respiratory, and reflected motions, when he endeavours to prove, that the reflected motions which take place after loss of the brain are not dependent on true sensation, but only on the centripetal nervous actions which take place in sensations. Sensation, will, motion, are the three links of the chain, when a motion is induced by pain; but if the middle link be destroyed, the connexion between the first and second with the consciousness ceases. We believe also that the reflected motions on stimuli of the skin, which take place after the removal of the brain, do not contain any proof that the stimulus excited true sensation in the spinal marrow; it is rather the centripetal conduction of the nervous principle which commonly takes place in sensations, but which here is no longer sensation, because it is no longer conducted to the brain, the organ of consciousness. During health also numerous reflected motions result from stimuli of the skin, which do not come as true sensations to the con-

* There may certainly be considerable doubt respecting the action of the cesophagus-a doubt which nothing but careful experiment can solve. But I think I have proof that the cardia closes and opens upon the principle of the reflex function, as well as the pharynx, and that it is under the influence of the internal excito-motory, or pneumogastric nerve.-M. H. 
sciousness, but still may excite violent impressions on the spinal marrow; as, for instance, the permanent contraction of the sphincters from the stimulus of the excrement and of the urine. But Dr. Marshall Hall goes too far, when he supposes that in health every motion on true sensation is induced by the will, and that all excitations of sensitive parts in the reflected motions are without sensation. For the reflected motions of sneezing, coughing, and many others follow actual sensations*.

"The reflected motions, and the involuntary not reflected motions are not to be confounded with one another. If the rima glottidis of an animal be touched, says Dr. Marshall Hall, a contraction takes place; the same, when the henrt is touched. By removal of the brain no alteration ensues; but if the medulla oblongata be removed, the contractions of the larynx on stimuli cease, while those of the heart continue. The action of stimuli on the heart is an immediate one dependent on its irritability; a stimulus applied to the larynx must, on the contrary, be propagated to the medulla oblongata, and the contraction results indirectly from it. In a snake after the removal of the head a motion of the larynx ensued; it was drawn downwards and closed, as soon as Dr. Marshall Hall touched a spot within the teeth of the lower jaw or the nasal apertures. After removal of the medulla oblongata this ceased. Lastly, he mentions as belonging to the reflex function, the winking of the eyelids when they are touched; the peculiar action on the respiration by tickling, or when cold water is thrown into the face; sneezing from stimuli of the nasal mucous membrane; cough; vomiting from stimuli of the larynx or pharynx; tenesmus from stimulation of the rectum; and strangury from that of the bladder. We see that the spasms in diseases may have very different sources. There are, for instance, spasmodic affections which have their seat in the motor nerves themselves, and others which have their cause in the brain and spinal marrow; but there are also reflected spasms, whose cause lies in stimulation of sensitive nerves, as those which often take place after intestinal stimulation, in dentition, odontalgia, and painful nervous affections from organic and inorganic lesions generally.

"The phenomena which we have now described, first from our own observations and then from those of Dr. Marshall Hall, have all this in common with one another, that the spinal

* These and other acts of the excito-motory system are attended by sensation, but are not the less independent of it; some are entirely without it. - M. H. 
marrow is the connecting link between a sensorial and a motorial motion of the nervous principle, though still the course which the conduction in the reflected motions from the sensitive to the notor nerves in the spinal marrow takes may be more definitely pointed out. The most common kind of reflected motion is, that the muscles of a limb, in which violent sensations have been excited, may be moved, as in the burning of the skin twitchings take place in the burned limb; or as in the commencement of the narcotization of an animal, on the sensitive stimulus of the skin the muscles of the stimulated limb are most easily moved; or as the morsels of food produce the reflected mction of the apparatus for swallowing; or as the particle in the conjunctiva exciting merely sensation, produces the reflected closure of the eyelids; or as, lastly, the stimulus of the urine and excrement act indirectly on the motion of the sphincters. As soon therefore as the sensitive-motion has reached the spinal marrow, it does not pass over the whole spinal marrow, but most easily to those motor nerves which have their origin nearest to the stimnlated sensitive nerves; or in other words, the easiest way for the current or vibration is from the posterior root of a nerve or some of its primitive filaments to its anterior root, or to the anterior roots of several adjacent nerves. We see, then, that the nervous principle in these currents or vibrations takes the shortest way, acting from sensitive fibres through the medulla spinalis on motor fibres; just as electricity takes the shortest way from one pole to the other. More correctly expressed, and translated into physiological language, this means, that in violent excitation of the motor property of the spinal marrow through a sensitive nerve, that part only of the spinal marrow is first excited, and then excites movements, which gives origin to the sensitive nerve; and that the excitation of other parts of the spinal marrow, and the motor nerves arising therefrom, decreases in proportion as they are more removed from the spot excited by the sensitive nerve. The same holds also of the cerebral nerves, whose reflected phenomena appear to remain still. almost quite unknown to Dr. Marshall Hall*. The great nerves of the senses are especially prone to cause reflected motions of the motor cerebral nerves, and especially the optic and auditory; they produce in vivid light and on lond sound a reflected excitation of the facial nerve, and thereby closure or winking of the eyelids. The optic nerve again easily produces the reflected excitation of the oculo-motor nerve in motion of the iris, and on looking at bright light it in-

* I am still of opinion that the reflex function is confined to the medulla oblongata and spinalis, exclusively of the brain.-M. H. 
duces a reflected affection of the facial and other nerves in sneezing. But the great sensitive nerve of the anterior part of the head and the face, the great portion of the trigeminus, may excite the oculo-motor and facial through the medium of the brain; thus contraction of the iris takes place when cold water is thrown into the nose, and from lickling in the nose sneezing takes place, as well as the action of the facial nerve in the excitement of the facial muscles which is connected with it. In short, we see, that of the motor cerebral nerves, the part of the oculo-motor nerve which goes to the ciliary ganglion and thence to the iris, and the facial nerve, may most easily be excited by reflexion, and that the impressions either of sight, or touch, or hearing may be the exciting causes: therefore between the origins of the optic, trigeminus, and auditory nerves, and the points of origin of the motor nerves in the brain, there nust be a facility of conduction pre-established by primitive formation. Those sensitive and motor nerves, whose mutual action is effected through the brain and spinal marrow, present a kind of balance with those central parts, one altering the other, as the ascent of one scale induces the descent of the other, or as the falling of a fluid in one leg of a bent tube produces the ascent in the other, till they are permanently at an equilibrium. If a sensitive nerve is not usually in a state to produce a reflected motion, yet on any violent impression on sensation it becomes so, and the brain and spinal marrow then reflect the currents or vibrations received from the sensitive nerves, into those motor nerves, to which the conduction from the sensitive fibres through the fibres of the brain and spinal marrow is most easy.

"Another very conımon path of conduction from the sensitive to the motor nerves through the medium of the spinal marrow and medulla oblongata, is that seen in the excitation of the mucous membranes and the secondary affection of the respiratory muscles in vomiting, evacnation of fæces, parturition, the coughing, sneezing, \&c. Next to the above-mentioned law, that nerves of allied origins, or of not very remote origins, are peculiarly prone to the phenomena of reflexion, the most frequently acting law of the "Nervenstatik" is the reflexion now mentioned. "Therefore, in the medulla oblongata and spinal marrow, between the sensitive nerves of the mucous membranes (the trigeminus in the nose; the vagus in the trachea, lungs, pharynx, œesophagus, stomach; the sympathetic in the intestinal canal and uterus; branches of the sacral plexus and sympathetic in the urinary bladder and rectum;) and the motor respiratory nerves (facial, accessory, and spinal nerves), there must be pre-formed easy means for a conduction; while, on 
the contrary, the spinal nerves going to the extremities are excluded from this harmony.

"But if a certain irritation of the spinal chord and brain be induced by narcotism or other causes, then every perception may produce a discharge of the spinal marrow to all the motor nerves, even to those which are affected with the greatest difficulty, viz. the motor nerves of the extremities." (pp. 688 -701.)

Such is the account of this subject given by Prof. Müller. I may be allowed to repeat that I have perused this unprejudiced and independent testimony to the importance of my investigations with unmingled satisfaction.

Before I dismiss the subject, I must add that my views are somewhat different from those of Prof. Müller:

1. I view the reflex function as the distinct and peculiar or proper function of the medulla spinalis, equally independent of the brain, the sympathetic, and of the anastomoses and the mere origins of nerves;

2. I regard this function as residing in the medulla, as the axis of a distinct system of excitor and motor, and excito-motory nerves;

3. I consider this function and its system of nerves as presiding over the orifices and the exits or sphincters of the animal frame, and over ingestion and egestion;

4. The brain is the central organ of sensation and volition, the organ of mental relation with the external world; the spinal marrow, on the contrary, is the central organ of excito-motory phenomena, and of the physical appropriation of certain external oljects ;

5. Respiration even is a part of this peculiar function : it is excited on ordinary, and on extraordinary occasions, through appropriate excitor nerves, especially the pneumo-gastric, but also the fifth and spinal nerves;

6. Volition may modify the acts of the reflex function, and these acts may be attended by sensation; but this function is, otherwise, independent both of rolition and sensation, of their organ the brain, and of the mind or soul;

7. The passions, in an especial manner, demonstrate themselves through the medium of the true spinal marrow; and thus pain may induce surprise or fear, and appear to occasion an excito-motory act ;

8. The brain sleeps; but the spinal marrow never sleeps;

9. Finaliy, the excito-motory system of nerves are the peculiar seat of action of certain diseases, and of certain causes and remedies of disease.

'These and other propositions 1 am about to illustrate in a 
series of papers preparing for the Royal Society. That they involve a principle in physiology at once extensive and novel, will not, I think, be now denied.

I may add that in some of the Invertebrata, the necessity for the nerves being intervertebral not existing, the excito-motory system of nerves, with their axis, may be as distinct in their anatomy as they are in their functions. This question I am about to subject to the test of experiment.

14, Manchester Square,

November 25,1836 .

XLIII. An Analysis of Dr. Ritchie's Paper, in reply to Mr. Rainey's last Communication concerning Magnetic Reaction, contained in the Philosophical Magazine for January. By G. RAINY, M.R.C.S.*

THAT Dr. Ritchie is in error will be clear from the following facts. 'The expression most frequently employed to explain the action of a magnet upon its keeper is, that the magnet induces on the contiguous ends of the armature opposite states of magnetism, and that in consequence of this dissinilarity these oppositely magnetized extremities at tract one another, evidently inferring that attraction follows as a consequence of induction. If a piece of soft iron be applied to magnets of different magnetic intensities, the adhesion would most uncioubtedly be the greatest between the soft iron and the strongest magnet; and if any other magnet still strongerwere applied to the same keeper, this increase would be manifested by a still more forcible attraction of this keeper by the magnet; and so on, without any known limit : consequently, as attraction is the effect of induction, the induction may be inferred to have no known limit, and the position to be true.

Dr. Ritchie says, " he, Mr. Rainey, also takes for granted, that a magnet having double the power will induce in the same armature twice the effect." I am not conscious of ever having made such an assertion.

Dr. Ritchie, to refute my first position and the one incorrectly attributed to me, has invented the following experiment:

"Roll a covered wire about the half of an electro-magnet A B. Do the same with an equal wire from $\mathrm{C}$ to $\mathrm{D}$. Connect the first helix with an elementary battery, and ascertain the lifting power of the magnet. Connect the other helix with an equal battery, and instead of the lifting power being doubled, according to the principle assumed by Mr. Rainey, its power

* Communicated by the Author.

Third Series. Vol. 10. No. 60. March 1837. 2 C 